\title{
The effect of NMDA-R antagonism on simultaneously acquired local field potentials and tissue oxygen levels in the brains of freely-moving
}

\section{rats}

\author{
John Kealy ${ }^{\text {a, c, }}$, Sean Commins ${ }^{\text {b }}$, John P. Lowry ${ }^{a}$ \\ a Department of Chemistry, Maynooth University, Maynooth, Co. Kildare, Ireland \\ b Department of Psychology, Maynooth University, Maynooth, Co. Kildare, Ireland \\ ' Smurfit Institute of Genetics, Trinity College Dublin, Dublin 2, Ireland
}

\section{A R T I C L E I N F O}

\section{Article history:}

Received 3 August 2016

Received in revised form

7 December 2016

Accepted 9 January 2017

Available online 11 January 2017

\section{Keywords:}

Ketamine

MK-801

Phencyclidine

Hippocampus

Striatum

High-frequency oscillations

\begin{abstract}
A B S T R A C T
Non-competitive NMDA receptor antagonists are known to induce psychosis-like symptoms in rodents. Administration of such compounds cause behavioural effects such as memory impairment and hyperlocomotion. Additionally, drugs such as phencyclidine (PCP), ketamine and MK-801 all cause distinctive increases in striatal local field potential (LFP) in the high frequency oscillation (HFO) band in the power spectrum (140-180 Hz). Amperometric sensors provide a means to measure tissue oxygen ( $\mathrm{tO}_{2}$; a $\mathrm{BOLD}^{-}$ like signal) in the brains of freely-moving rats while simultaneously acquiring LFP using the same electrode. Carbon paste electrodes were implanted into the striatum and hippocampus of male Wistar rats. Rats were administered with saline, ketamine (10 mg/kg), MK-801 (0.1 mg/kg) and PCP (2.5 mg/kg) and recordings were made at $1 \mathrm{kHz}$ using three different potentials ( $-650 \mathrm{mV}$ to measure $\mathrm{tO}_{2} ; 0 \mathrm{mV}$ and $+700 \mathrm{mV}$ as control conditions). NMDA receptor antagonism caused significant increases in $\mathrm{tO}_{2}$ in both the striatum and the hippocampus. Power spectrum analysis showed significant increases in HFO power in the striatum but not in the hippocampus. Conversely, there were significant decreases in delta and alpha power along with increases in theta and gamma power in the hippocampus that were absent in the striatum. This supports findings that LFP can be obtained from an amperometric sensor signal; allowing simultaneous acquisition of two translational biomarkers of neuronal activity (LFP and tO $\mathrm{L}_{2}$ ).
\end{abstract}

(c) 2017 Elsevier Ltd. All rights reserved.

\section{Introduction}

The $N$-methyl-D-aspartate glutamate receptor (NMDA-R) is firmly implicated in normal physiological processes such as synaptic plasticity and learning along with being implicated in diseased and disordered states such as excitotoxicity and schizophrenia. Indeed, non-competitive NMDA-R antagonists are routinely used to model schizophrenia in rodents (Neill et al., 2014; Janhunen et al., 2015). Additionally, given the use of noncompetitive NMDA-R antagonists in humans as medical interventions (ketamine) and as drugs of abuse (phencyclidine; PCP), it is important to understand the neurophysiological effects of these drugs to find new clinical uses for existing drugs like

\footnotetext{
* Corresponding author. Smurfit Institute of Genetics, Trinity College Dublin, Dublin 2, Ireland.

E-mail address: kealyjo@tcd.ie (J. Kealy).
}

ketamine and to design novel antipsychotic and antidepressant compounds with fewer side effects and greater therapeutic value.

In rodents, it has been demonstrated that non-competitive NMDA-R antagonists can induce characteristic changes in electrophysiological measurements in various brain regions. The three classic non-competitive antagonists, ketamine, PCP and MK-801, all alter multiple frequencies of the local field potential (LFP) signal in multiple regions in a dose-dependent manner (for a comprehensive review, see Hunt and Kasicki, 2013). Notably, these drugs are associated with increases in striatal high-frequency oscillations (HFOs; Hunt et al., 2006, 2011) and in hippocampal gamma power (Ma and Leung, 2000; Ehrlichman et al., 2009; Lazarewicz et al., 2010; Hunt et al., 2011; Saunders et al., 2012).

Attempts at using functional magnetic resonance imaging (fMRI) to directly compare changes in brain activity between animals and humans have been made but are limited by the need to anaesthetise or sedate animals, thus introducing a confounding factor when interpreting data (Hodkinson et al., 2012). Even so, 


\begin{tabular}{|ll|}
\hline Abbreviations \\
BOLD & Blood-oxygen-level-dependent \\
CPE & Carbon paste electrode \\
FFT & Fast Fourier transform \\
fMRI & Functional magnetic resonance imaging \\
HFO & High-frequency oscillation \\
LFP & Local field potential \\
NMDA-R & N-methyl-D-aspartate glutamate receptor \\
PCP & Phencyclidine \\
SCE & Saturated calomel electrode \\
tO2 & Tissue oxygen \\
\hline
\end{tabular}

pharmacological MRI studies in rodents show that non-competitive NMDA-R antagonists are associated with increased regional cerebral blood volume in the striatum (Gozzi et al., 2008a, 2008b; Broberg et al., 2013) and hippocampus (Gozzi et al., 2008a, 2008b) as well as increases in the blood-oxygen-level-dependent (BOLD) signal in the striatum and hippocampus (Littlewood et al., 2006; Chin et al., 2011).

Therefore, it is desirable to develop techniques that offer greater translational validity to make better-informed interpretations of data generated in animal models. Amperometry is of particular interest as it is possible to measure changes that are comparable to imaging techniques routinely used in humans (BOLD and EEG; see Lowry et al., 2010; Zhang et al., 2009) and can be used in freelymoving animals With electrochemical sensors, it is possible to measure real-time changes in tissue oxygen $\left(\mathrm{tO}_{2}\right)$ in the brain with high spatial resolution (Bolger and Lowry, 2005; Bolger et al., 2011a, 2011b) and this $\mathrm{tO}_{2}$ signal correlates highly with changes in the BOLD signal acquired simultaneously (Lowry et al., 2010). This permits researchers to utilise $\mathrm{tO}_{2}$ measurements as a surrogate for fMRI in rodents, allowing for pharmacological (Kealy et al., 2013, 2015) and behavioural (McHugh et al., 2011) studies to be performed in awake rodents.

Furthermore, it has been demonstrated by recording LFP while simultaneously measuring changes in choline using an amperometric sensor that the amperometric signal is made up of a low frequency component $(<1 \mathrm{~Hz})$ corresponding to neurochemical changes and a higher frequency component $(>1 \mathrm{~Hz})$ corresponding to LFP (Zhang et al., 2009). The aim of this study is to confirm that the signal obtained from oxygen sensors can also be broken down into a low frequency component corresponding directly to changes in oxygen and a higher frequency component that corresponds to changes in LFP. In order to test this hypothesis, we use ketamine, PCP, and MK-801 to pharmacologically determine whether the $>1 \mathrm{~Hz}$ components of the $\mathrm{tO}_{2}$ signal are in fact changes in LFP. As these drugs all induce known changes in the LFP (Hunt and Kasicki, 2013), we can assume that if the higher frequency components of the $\mathrm{tO}_{2}$ signal are in fact related to the LFP, they will change in a similar manner. Additionally, baseline activity and the effects of changing oxygen levels in vivo along with control experiments in vitro will be examined in a similar manner to help determine the relationship between $\mathrm{tO}_{2}$ and LFP as translational markers of neuronal activity akin to local and scalp electrophysiological recordings made in humans. Taken together, these findings will demonstrate how amperometry can be used to measure changes in $\mathrm{tO}_{2}$ and LFP simultaneously, allowing for detailed translational assessments of neuropharmacological and behavioural interventions to be performed.

\section{Materials and methods}

\subsection{Subjects}

Male Wistar rats (250-300 g; Charles River Laboratories International, Inc.; U.K.) were housed in a temperature-controlled facility with a 12-h light/dark cycle (lights on at 08:00) with access ad libitum to food and water. All procedures were performed under license in accordance with the European Commission Directive 2010/63/EU and were approved by the Maynooth University Ethics Committee.

\subsection{Data acquisition and statistical analysis}

All electrochemical experiments were performed using a low noise potentiostat (Quadstat, eDAQ, Australia). Data acquisition was performed at $1 \mathrm{kHz}$ using an eCorder ${ }^{\circledR}$ interface system and eDAQ Chart software (eDAQ, Australia). Electrophysiological recordings were made using a Micro1401 mk II data acquisition unit (Cambridge Electronic Design, U.K.) and Spike2 software (CED, U.K.). Electrophysiological recordings were made at $10 \mathrm{kHz}$ and the signal was amplified and filtered between $0.1 \mathrm{~Hz}$ and $1 \mathrm{kHz}$ using a DAM50 amplifier (WPI, U.S.A.). Data was preliminarily processed in Microsoft ${ }^{\circledR}$ Excel $^{\circledR} 2010$ before being exported to GraphPad Prism ${ }^{\circledR}$ 5.01 for statistical analysis. Data was either normalised to baseline levels or area under the curve (AUC) analysis was performed to quantify any observed changes in the sensor signals over time for statistical analysis. Fast Fourier transforms (FFTs) were performed on 1-min time bins in eDAQ Chart and resulting power spectra were split into five frequency bands (delta, $1-4 \mathrm{~Hz}$; theta, $5-8 \mathrm{~Hz}$; alpha, 9-12 Hz; gamma, 30-100 Hz; HFO, 140-180 Hz). For multiple comparisons, repeated-measures and mixed-factorial analysis of variance tests (ANOVAs) with Bonferroni post-hoc analysis were used as appropriate. $p<0.05$ was considered to be significant.

\subsection{Working electrode preparation and surgery}

Carbon paste electrodes (CPEs; O'Neill et al., 1982) were prepared and in vitro calibrations were performed using constant potential amperometry in a standard three-electrode glass electrochemical cell containing $20 \mathrm{ml}$ PBS at room temperature using a saturated calomel electrode (SCE) as the reference electrode and a bare platinum wire as the auxiliary electrode (see Lowry et al., 1997; Kealy et al., 2013, 2015). For CPE $\mathrm{O}_{2}$ calibrations, a three-point calibration protocol was used $(0,240,1200 \mu \mathrm{M})$ using an applied potential of $-650 \mathrm{mV}$ versus SCE. The current was recorded throughout the course of each calibration and analysis was performed using quiescent steady-state conditions (Kealy et al., 2013, 2015).

Animals were anaesthetised using isofluorane (4\% for induction, 1.5-3.0\% for maintenance; IsoFlo ${ }^{\mathbb{R}}$, Abbott, U.K.) and placed in a stereotaxic frame. The skull was exposed and four screws were implanted into the skull with one doubling as the auxiliary electrode. Burr holes were made for the other electrodes. Two CPEs were implanted into each hemisphere, targeting the striatum (+1.7 mm A/P; $\pm 2.5 \mathrm{~mm} \mathrm{M/L;}-5.0 \mathrm{~mm} \mathrm{D/V})$ and dorsal hippocampus $(-4.0 \mathrm{~mm} \mathrm{~A} / \mathrm{P} ; \pm 3.0 \mathrm{~mm} \mathrm{M} / \mathrm{L} ;-2.5 \mathrm{~mm} \mathrm{D} / \mathrm{V})$. The silver reference electrode was implanted into the left parietal lobe. All electrodes were cemented into place (Dentalon ${ }^{\mathbb{B}}$ Plus, HeraeusKulzer, Germany) and the gold contacts at the end of each electrode were cemented into a six-pin Teflon ${ }^{\circledR}$ socket (Plastics One, U.S.A.). All animals were given saline ( $0.9 \% ; 3 \mathrm{ml} / \mathrm{kg}$ body weight) and perioperative analgesia was provided $(0.3 \mathrm{mg} / \mathrm{kg}$ body weight; 
Buprecare $^{\circledR}$, AnimalCare Ltd., U.K.) before animals were allowed to recover in an incubator.

\subsection{Recording procedure}

Following surgery, animals were housed in Raturn ${ }^{\circledR}$ sampling cage systems (BASi, U.S.A.). All experiments were performed in the animal's home bowl following five days of recovery. The sensors from each animal were connected to the potentiostat via the sixpin Teflon ${ }^{\circledR}$ socket using a flexible screened six-core cable (Plastics One, U.S.A.). Cabling was mounted on the Raturn ${ }^{\circledR}$ bowl to allow free movement of the animal. Movement was recorded in eDAQ Chart using an infrared motion detector.

Baseline recordings of $\mathrm{tO}_{2}$ (applied voltage: $-650 \mathrm{mV}$ ) were made daily in the striatum and hippocampus (Kealy et al., 2013). Animals were given intraperitoneal injections of saline (0.9\%); MK801 (0.1 mg/kg; Sigma, U.K.); phencyclidine hydrochloride (PCP; $2.5 \mathrm{mg} / \mathrm{kg}$; Department of Chemistry, Maynooth University) and (S)-(+)-ketamine hydrochloride (10 mg/kg; Tocris, Bio-Techne, U.K.). One injection was given per day with a washout period of $48 \mathrm{~h}$ between treatments. Treatments were given in a pseudorandom order to minimise interaction between the active compounds. Baseline recordings were made for $1 \mathrm{~h}$ before injections followed by a $2 \mathrm{~h}$ recording period. All animals were disconnected at night and housed together to minimise stress from being singly housed.

Recordings were also made at $0 \mathrm{mV}$ (where there should be minimal redox activity at the electrode surface; Bolger et al., 2011b) and at $+700 \mathrm{mV}$ (where ascorbic acid and other oxidisable species, but not oxygen, are detectable; Lowry et al., 1996). Finally, recordings were made using CPEs connected to a standard electrophysiology apparatus. CPEs implanted in the striatum of each animal were connected to the amplifier for the electrophysiological recording device via the same cabling and housing as above and changes in potential at the recording electrode were measured. For these recordings, only saline and $(\mathrm{S})-(+)$-ketamine hydrochloride injections were administered.

\section{Results}

\subsection{Power spectral analysis of the amperometric oxygen signal}

In order to determine whether the electrochemical reaction at the CPE active surface had any effect on frequency components $>1 \mathrm{~Hz}$, in vitro calibration data was analysed using FFT analysis at $0 \mu \mathrm{M} ; 240 \mu \mathrm{M}$ and $1200 \mu \mathrm{M}$ oxygen during quiescent periods in the oxygen calibration. There was an overall increase in power across all frequencies in the presence of oxygen compared to $0 \mu \mathrm{M}$ but no differences between $240 \mu \mathrm{M}$ and $1200 \mu \mathrm{M}$ oxygen (Fig. 1A). This indicates that once oxygen is present (as is the case in the brain), the reduction of oxygen at the electrode surface should not interfere with frequency components above $1 \mathrm{~Hz}$.

During baseline recording in vivo, movement was associated with increases in $\mathrm{tO}_{2}$ in both the striatum and the hippocampus (Fig. 1B). FFT analysis was performed on 2-min time bins of the oxygen signals recorded while animals were awake but at rest $(n=10)$ and while animals were engaged in exploratory movement $(n=10)$. In the striatum (Fig. 1C), there was a sharpening of the theta peak at $8 \mathrm{~Hz}$, an appearance of another peak at around $14-20 \mathrm{~Hz}$ and a broadband increase in gamma power (not related to the spike at $50 \mathrm{~Hz}$ resulting from mains noise). Paired t-tests comparing stationary and moving conditions (Fig. 1D) showed that there was a significant decrease in striatal alpha $(t=3.464 ; d f=9$; $p<0.01)$ and a significant increase in striatal gamma $(t=2.425$; $d f=9 ; p<0.05$ ). In the hippocampus (Fig. $1 \mathrm{E}$ ), there were similar changes compared to striatum. There was again a sharpening of the theta peak but at a slightly lower frequency $(7 \mathrm{~Hz}$ ) along with the appearance of another peak at around $14-17 \mathrm{~Hz}$ though there were no changes in gamma power. Paired t-tests (Fig. 1F) showed that there was a significant decrease in hippocampal alpha power $(t=2.751 ; d f=9 ; p<0.05)$.

To confirm that the changes observed in vivo are attributable to changes in LFP and not changes in $\mathrm{tO}_{2}$, the same movement experiments were repeated with applied potentials of $0 \mathrm{mV}$ and $+700 \mathrm{mV}$ where changes in $\mathrm{tO}_{2}$ should have no effect on the recorded currents. As such, there were no discernible changes in current associated with movement in either the striatum or the hippocampus at $0 \mathrm{mV}$ (Fig. 2A) or $+700 \mathrm{mV}$ (Fig. 2B). Power spectra at $0 \mathrm{mV}(\mathrm{n}=4)$ showed similar changes associated with movement as observed at $-650 \mathrm{mV}$ in both the striatum (Fig. 2C) and hippocampus (Fig. 2E). Similarly, at $+700 \mathrm{mV}$, power spectra $(\mathrm{n}=6)$ in the striatum (Fig. 2D) and hippocampus (Fig. 2F) were similar to those observed at 0 and $-650 \mathrm{mV}$.

\subsection{Effect of non-competitive NMDA receptor antagonists on local field potential and $\mathrm{tO}_{2}$}

Following a baseline period of $10 \mathrm{~min}$, animals were given an intraperitoneal injection on different days with either saline $(0.9 \%$; vehicle control) or a non-competitive NMDA-R antagonist (ketamine; PCP; MK-801). Recordings were made from the striatum and hippocampus for $2 \mathrm{~h}$ post-injection and analysed post hoc in order to extract the LFP components of the signals. Briefly, the oxygen signals from each animal were divided into 1-min time bins and FFT analysis was performed on them. The resulting power spectra were divided into five frequency bands, averaged and normalised to the baseline period. Changes in LFP power were plotted against time to compare with changes in $\mathrm{tO}_{2}$. Following saline administration $(\mathrm{n}=15$; Fig. $3 \mathrm{~A})$, there was a transient increase in movement and in $\mathrm{tO}_{2}$ in both the striatum and hippocampus associated with injection stress. Changes in LFP were similarly transient with increases in gamma power and decreases in theta and alpha power in the striatum along with increases in theta, gamma and HFO power and a decrease in delta power in the hippocampus.

Ketamine administration ( $\mathrm{n}=15$; Fig. $3 \mathrm{~B}$ ) induced hyperlocomotion lasting approximately $35 \mathrm{~min}$ with an accompanying increase in $\mathrm{tO}_{2}$ in both brain regions. These changes in $\mathrm{tO}_{2}$ were very similar but the changes in LFP showed regional differences with increases in gamma and HFO power and decreases in theta and alpha power in the striatum. Whereas in the hippocampus, there was an increase in gamma power and decreases in delta and alpha power associated with ketamine treatment.

MK-801 administration ( $\mathrm{n}=8$ for striatum, $\mathrm{n}=9$ for hippocampus; Fig. 3C) induced hyperlocomotion and an increase in $\mathrm{tO}_{2}$ in both the striatum and the hippocampus lasting at least $120 \mathrm{~min}$. In the striatum, there was a very large increase in HFO power lasting at least 120 min along with more moderate increases in delta and gamma power and decreases in theta and alpha power. In the hippocampus, there were increases in theta, gamma and HFO power along with a decrease in delta power. Alpha power showed a phasic response with an initial decrease (0-15 min) followed by an increase (15-40 $\mathrm{min}$ ) before dipping below baseline again (70-110 $\mathrm{min})$.

PCP administration ( $\mathrm{n}=6$ for striatum, $\mathrm{n}=7$ for hippocampus; Fig. 3D) induced hyperlocomotion lasting at least $120 \mathrm{~min}$. However, unlike ketamine or $\mathrm{MK}-801, \mathrm{tO}_{2}$ levels in both regions returned to baseline after approximately $45 \mathrm{~min}$ (i.e. before hyperlocomotion wore off). In the striatum, there was a large increase in HFO power and moderate increases in gamma power whereas theta and alpha power both decreased in power together. 
A

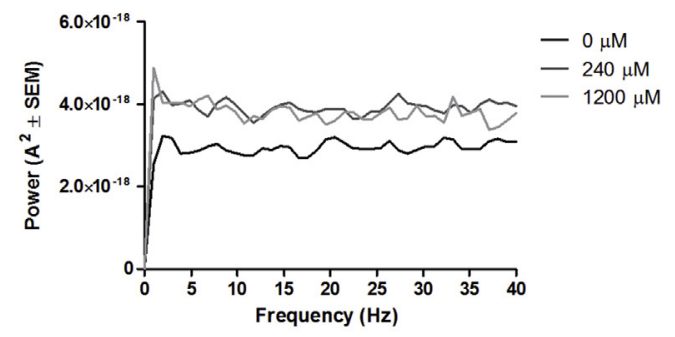

B

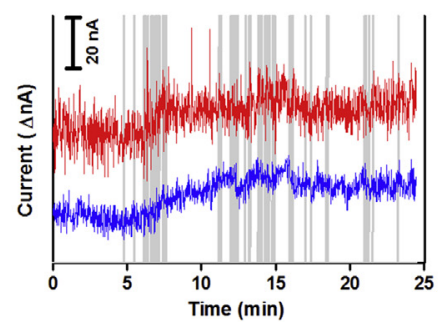

C
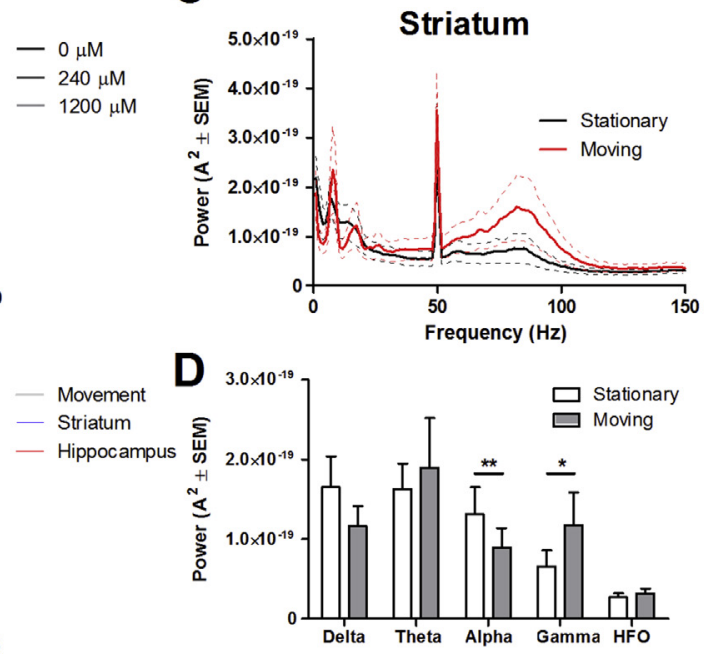

E
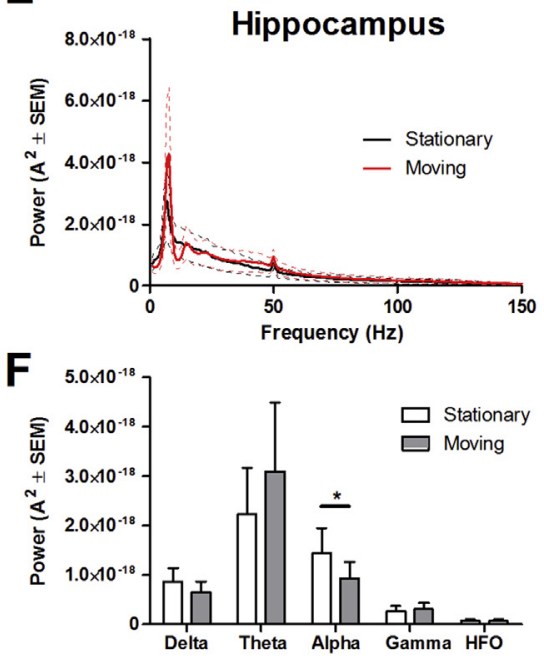

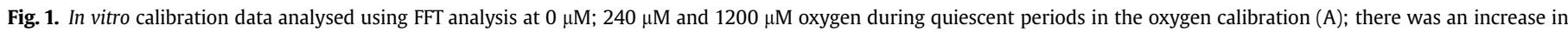

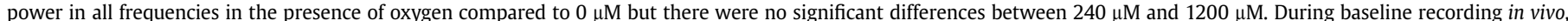

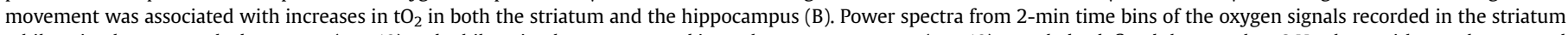

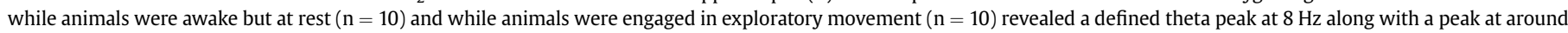

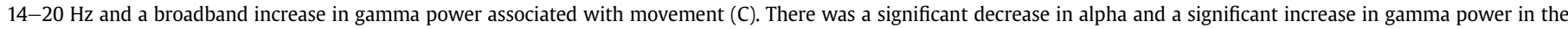

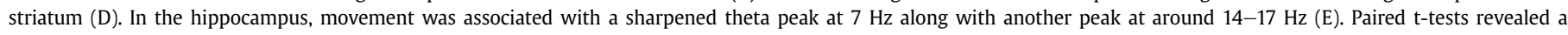
significant decrease in alpha power in the hippocampus (F). ${ }^{*} p<0.05 ;{ }^{* *} p<0.01$; ${ }^{* * *} p<0.001$.
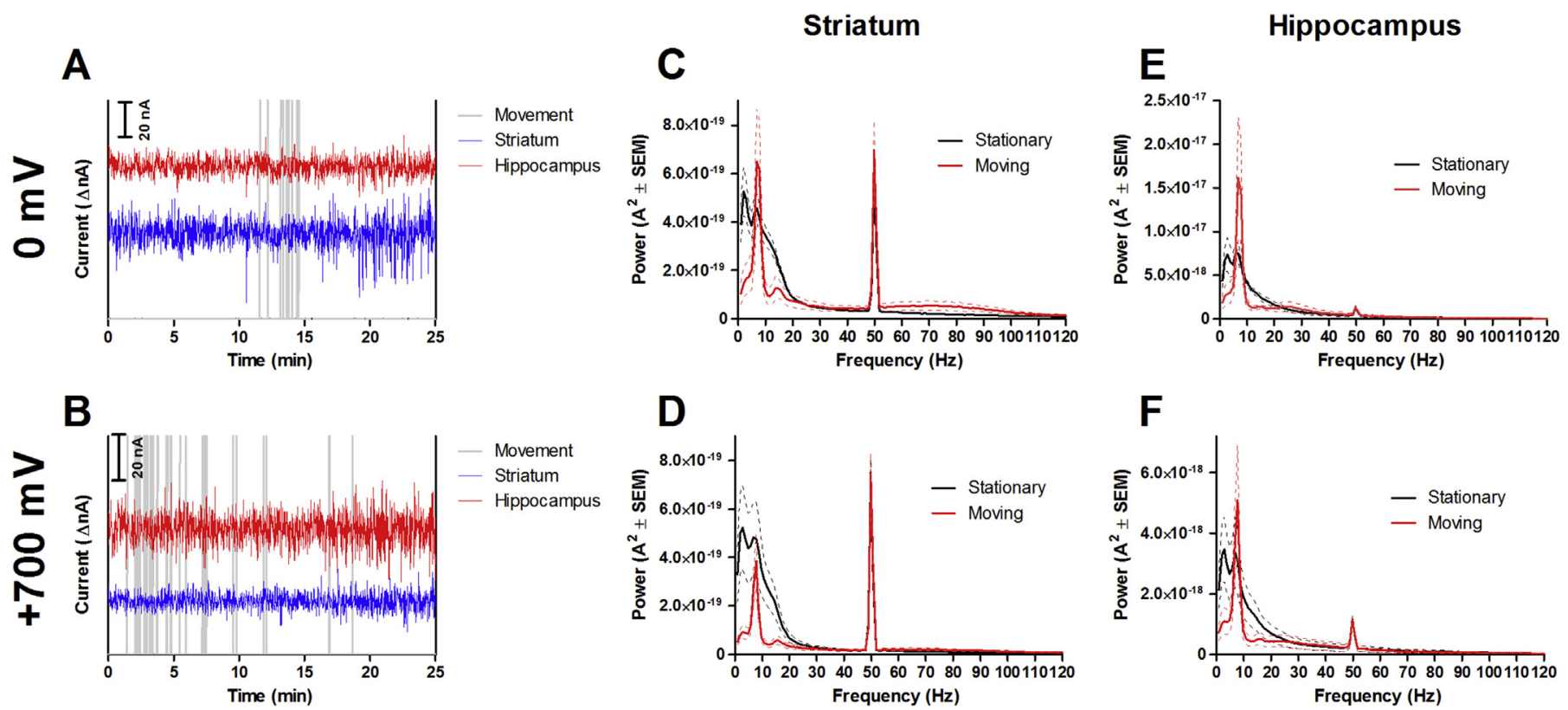

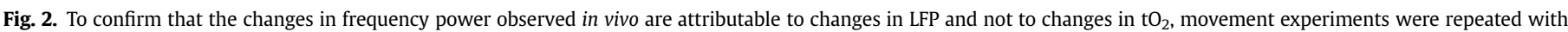

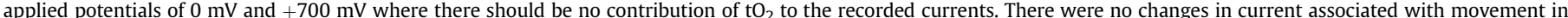

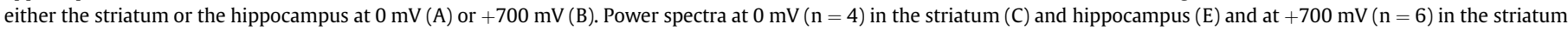
(D) and hippocampus (F) showed similar changes associated with movement as the power spectra obtained at $-650 \mathrm{mV}$.

In contrast to MK-801, there was a decrease in striatal delta. In the hippocampus, there was an increase in gamma power lasting at least 120 min though unlike MK-801, both theta and HFO power remained at baseline levels throughout the recording period. Delta and alpha power both showed a phasic response to PCP treatment with an initial decrease $(0-25 \mathrm{~min})$ followed by an increase (40-60 $\mathrm{min}$ ) before dipping below baseline again (80-120 min).

In order to compare the effect of each drug treatment to each other, AUC analysis was performed on baseline-corrected data from the 30-min period immediately following injection and two-way ANOVA was used to make comparisons between the effects seen due to drug treatment and brain region on $\mathrm{tO}_{2}$ and LFP data. For $\mathrm{tO}_{2}$ recordings (Fig. 4A), there was a significant effect for drug treatment $(F=10.70 ; d f=3,69 ; p<0.001)$ but there was no effect for brain region $(F=0.6383 ; d f=1,69 ; p>0.05)$ and there was no interaction effect $(F=1.489 ; d f=3,69 ; p>0.05)$. Bonferroni post 

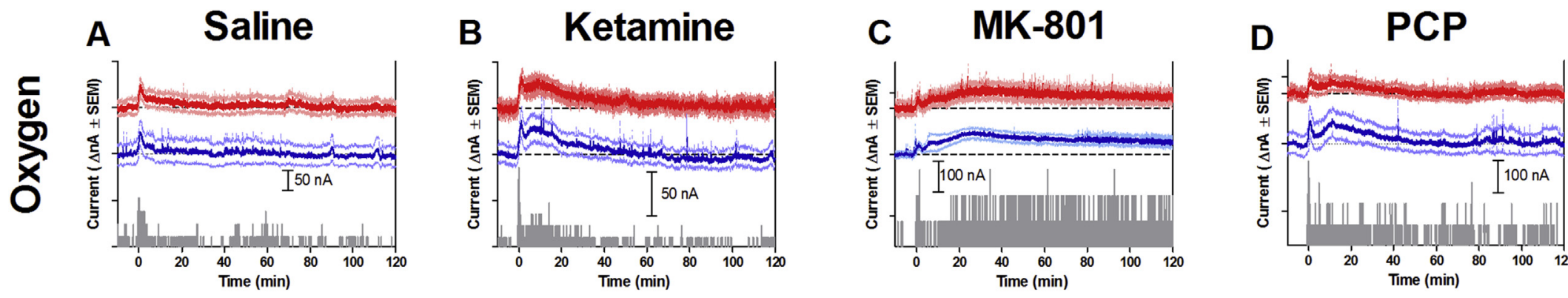

- Striatum

- Hippocampus

- Movement
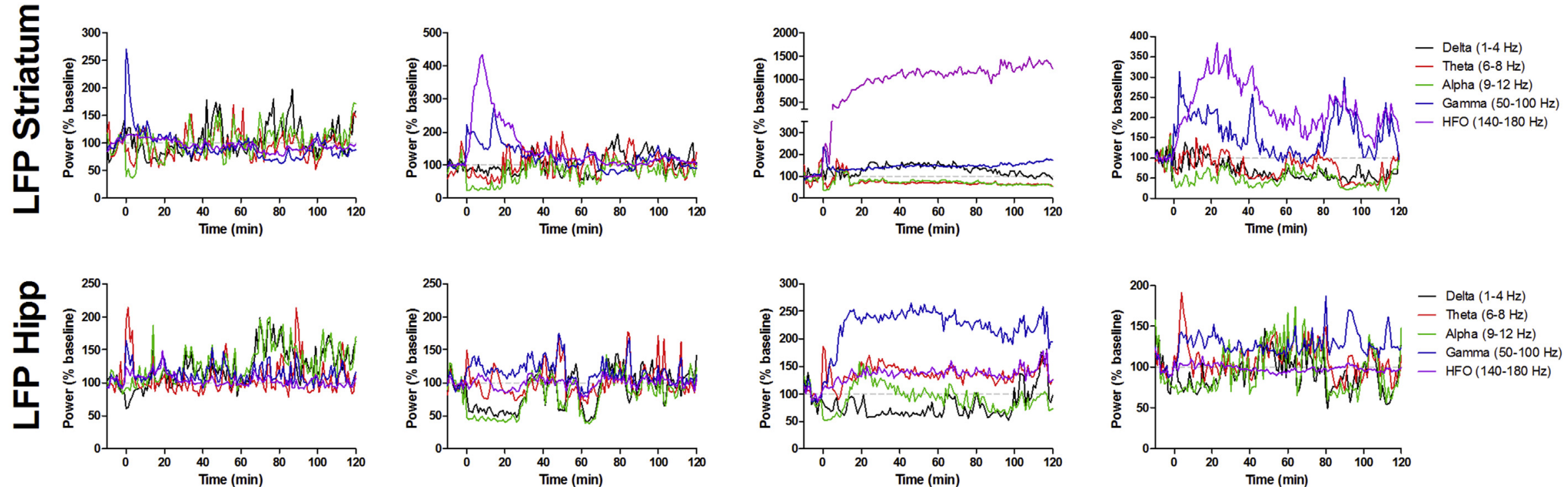

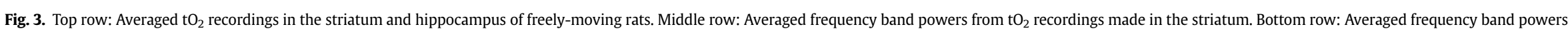
from $\mathrm{TO}_{2}$ recordings made in the hippocampus. Changes in frequency band powers shown without SEM for clarity. Following a baseline period of 10 min, animals were given an intraperitoneal injection containing either saline ( $0.9 \%$; vehicle control) or a non-competitive NMDA-R antagonist (ketamine; PCP; MK-801). Following saline administration $(\mathrm{n}=15 ; \mathrm{A})$, there was a transient increase in movement along with an increase in tO ${ }_{2}$ levels in both the striatum and hippocampus accompanied by changes in theta, alpha and gamma power in the striatum and in delta, theta, gamma and HFO power in the hippocampus. Ketamine administration ( $\mathrm{n}=15$; $\mathrm{B}$ ) induced hyperlocomotion with an accompanying increase in $\mathrm{tO}_{2}$ in both brain regions. Changes in LFP showed regional differences with increases in gamma and HFO power and decreases in theta and alpha power in the striatum compared to increases in gamma power and decreases in delta and alpha power in the hippocampus. MK-801 administration ( $\mathrm{n}=8$ for striatum, $\mathrm{n}=9$ for hippocampus; $\mathrm{C})$ induced hyperlocomotion and an increase in tO ${ }_{2}$ in both brain regions. In the striatum, there was a

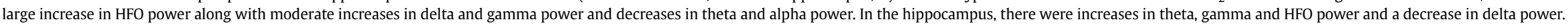
PCP administration ( $\mathrm{n}=6$ for striatum, $\mathrm{n}=7$ for hippocampus; $\mathrm{D}$ ) induced hyperlocomotion and increased $\mathrm{tO}_{2}$ levels in both brain regions. In the striatum, there was a large increase in $\mathrm{HFO}$ power, moderate increases in gamma power and decreases in delta, theta and alpha power. In the hippocampus, there was an increase in gamma power and a phasic response was observed in delta and alpha power. 
A
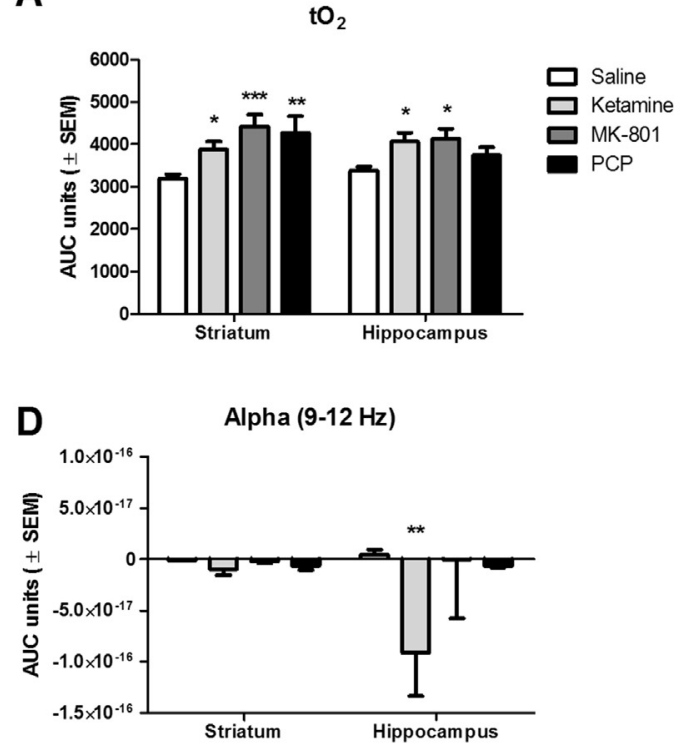

B

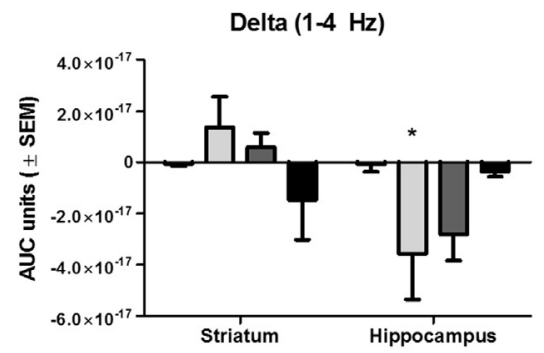

E

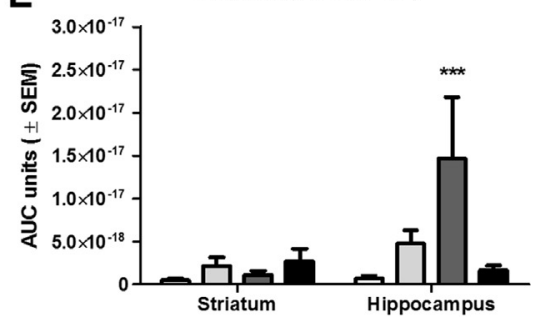

C

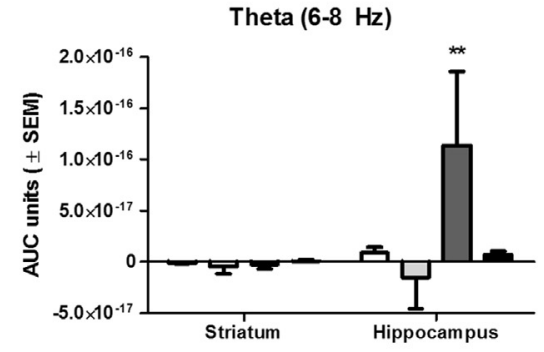

$\mathbf{F}$

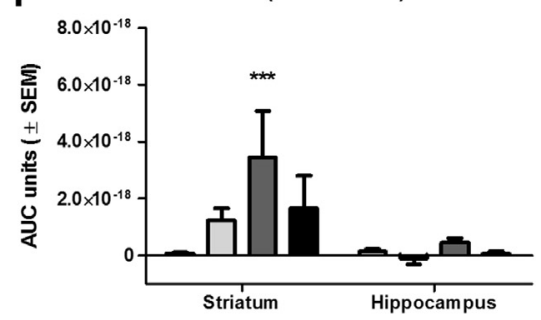

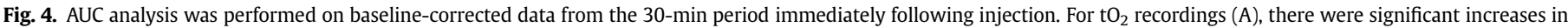

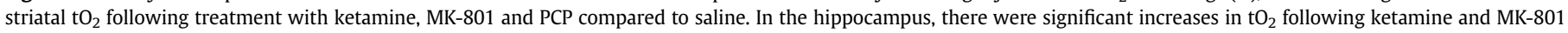

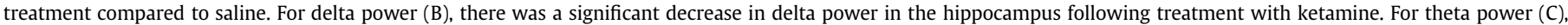

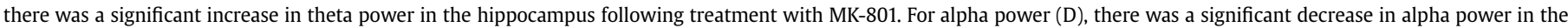

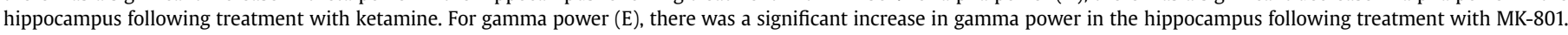
For HFO power (F), there was a significant increase in HFO power in the striatum following treatment with MK-801. ${ }^{*} p<0.05$; ${ }^{* *} p<0.01$; ${ }^{* * *} p<0.001$.

hoc tests showed that there were significant increases in striatal $\mathrm{tO}_{2}$ following treatment with ketamine $(p<0.05)$, MK-801 $(p<0.001)$ and PCP $(p<0.01)$ compared to saline. In the hippocampus, there were significant increases in $\mathrm{tO}_{2}$ following ketamine $(p<0.05)$ and MK-801 treatment $(p<0.05)$ compared to saline.

For delta power (Fig. 4B), there was no significant effect for drug treatment $(F=0.5499 ; d f=3,59 ; p>0.05)$ but there was a significant effect for brain region $(F=5.176 ; d f=1,59 ; p<0.05)$ along with a significant interaction effect $(F=3.551 ; d f=3,59 ; p<0.05)$. Bonferroni post hoc tests showed that there was a significant decrease in delta power in the hippocampus following treatment with ketamine $(p<0.05)$. For theta power (Fig. $4 \mathrm{C})$, there were no significant effects for drug treatment $(F=2.440 ; d f=3,62$; $p>0.05)$ or brain region $(F=2.914 ; d f=1,62 ; p>0.05)$, nor was there an interaction effect $(F=2.394 ; d f=3,62 ; p>0.05)$. Bonferroni post hoc tests showed that there was a significant increase in theta power in the hippocampus following treatment with MK-801 $(p<0.01)$. For alpha power (Fig. 4D), there were no significant effects for drug treatment $(F=2.473 ; d f=3,62 ; p>0.05)$ or brain region $(F=1.066 ; d f=1,62 ; p>0.05)$, nor was there an interaction effect $(F=1.759 ; d f=3,62 ; p>0.05)$. Bonferroni post hoc tests showed that there was a significant decrease in alpha power in the hippocampus following treatment with ketamine $(p<0.01)$.

For gamma power (Fig. $4 \mathrm{E}$ ), there were significant effects for drug treatment $(F=4.055 ; d f=3,66 ; p<0.05)$ and brain region $(F=6.407 ; d f=1,66 ; p<0.05)$, as well as an interaction effect $(F=4.005 ; d f=3,66 ; p<0.05)$. Bonferroni post hoc tests showed that there was a significant increase in gamma power in the hippocampus following treatment with MK-801 $(p<0.001)$. For HFO power (Fig. 4F), there were significant effects for drug treatment $(F=3.142 ; d f=3,69 ; p<0.05)$ and brain region $(F=10.25 ; d f=1$, $69 ; p<0.01)$, but there was no interaction effect $(F=2.163 ; d f=3$, $69 ; p>0.05)$. Bonferroni post hoc tests showed that there was a significant increase in HFO power in the striatum following treatment with MK-801 $(p<0.001)$.

To confirm that changes in these frequency bands were not related to changes in $\mathrm{tO}_{2}$, an applied potential of $0 \mathrm{mV}$ was used. There was no change in current following ketamine administration, indicating that there was no contribution of $\mathrm{tO}_{2}$ to the $0 \mathrm{mV}$ signal (Fig. 5A). At $0 \mathrm{mV}$, there was a similar change in frequency bands associated with LFP as observed at $-650 \mathrm{mV}$ including distinctive increase in HFO power in the striatum (Fig. 5B) and an increase in gamma power in the hippocampus (Fig. 5C). Finally, CPEs implanted in the striatum were connected to a traditional electrophysiological recording device and voltage changes were recorded with no potential applied to the CPE at all (Fig. 5D). Ketamine administration showed similar changes in power as it did when a voltage was being applied to the sensor, including an increase in HFO power (Fig. 5E).

\section{Discussion}

This study confirms that recordings made with amperometric sensors contain two main components: a slow, chemical-based component (in this case attributable to oxygen) and a faster, electrophysiological-based component (attributable to LFP; Zhang et al., 2009). Evidence to support this finding comes from the lack of contribution of oxygen to frequency components above $1 \mathrm{~Hz}$ in vitro and the similarity of higher frequency components of the amperometric signal recorded in vivo to recordings made with standard electrophysiological protocols (as presented here and in the wider literature). Using amperometric sensors in such a way allows for increased translational validity as changes in $\mathrm{tO}_{2}$ can be used as an analogue of BOLD activity in fMRI (Lowry et al., 2010) while simultaneously acquiring electrophysiological information that can be related to human electrophysiological recordings. This enables preclinical researchers to design experiments in freelymoving animals that better approximate human neuroimaging studies (Francois et al., 2012, 2014; 2016), thus providing preclinical researchers a powerful tool for understanding the relationships between brain activity with neuropharmacological and behavioural interventions. 
A

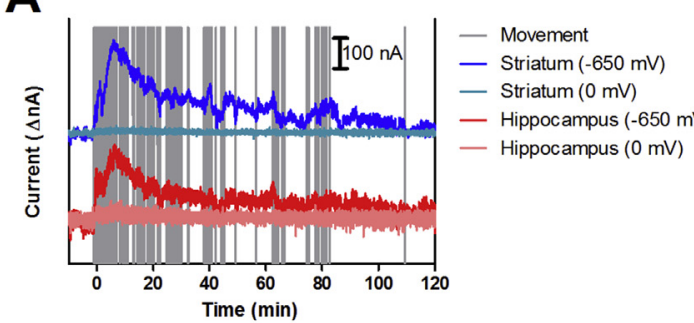

B

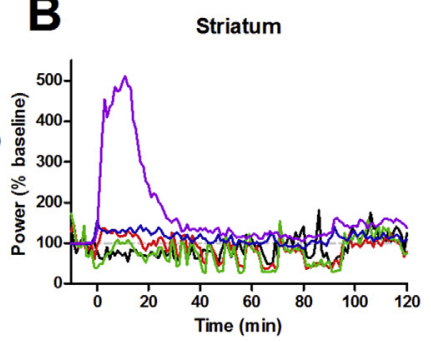

D
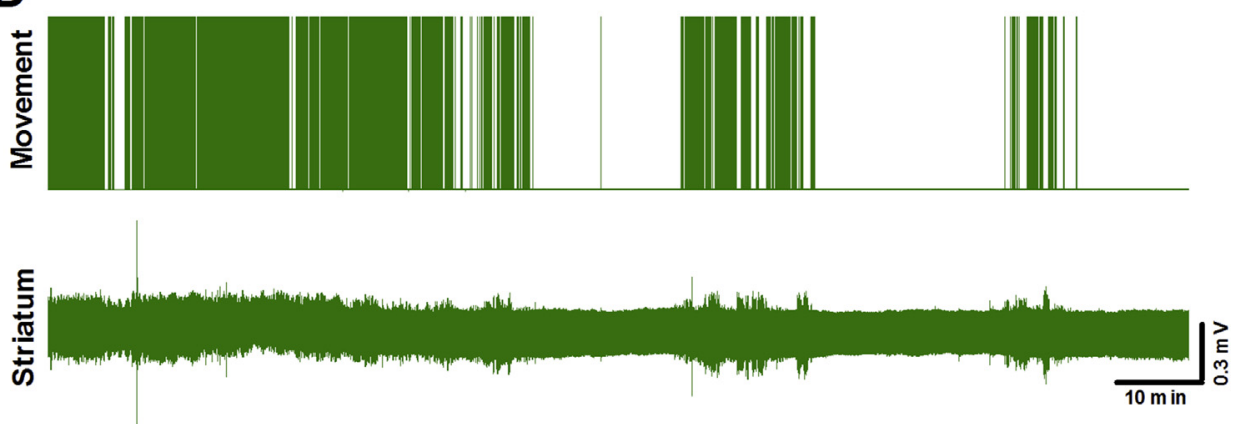

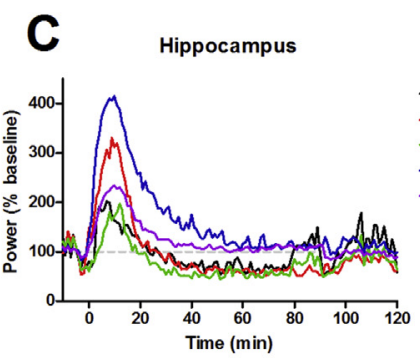

- Delta $(1-4 \mathrm{~Hz})$

- Theta $(6-8 \mathrm{~Hz})$

- Alpha $(9-12 \mathrm{~Hz})$

- Gamma $(50-100 \mathrm{~Hz})$

- HFO $(140-180 \mathrm{~Hz})$

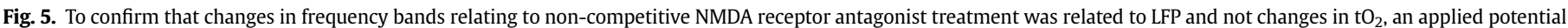

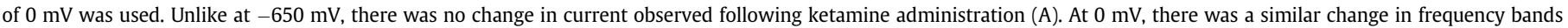

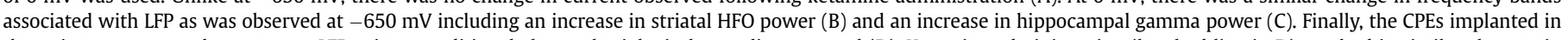

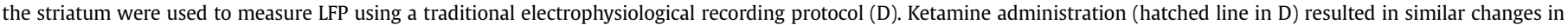
frequency bands associated with LFP power as it did when a voltage was being applied to the CPE, including an increase in HFO power (E).

By applying this method of simultaneously recording $\mathrm{tO}_{2}$ and LFP, we demonstrate here the pharmacological effects of three noncompetitive NMDA-R antagonists on neuronal activity in the rat brain. These compounds were used as they have been consistently shown to modulate LFP in rodents in a known way (see Hunt and Kasicki, 2013 for an overview). As was expected, pharmacologicallu induced changes in $\mathrm{tO}_{2}$ described here align with previous work with non-competitive NMDA-R antagonists using amperometry (Finnerty et al., 2013) and pharmacological MRI (Li et al., 2014). Importantly, many of the corresponding changes in power that were found in the LFP component of the amperometric signal correspond with changes that been reported in the wider electrophysiological literature. In the striatum, there were significant increases in HFO power following MK-801 treatment, similar to those seen in standard electrophysiology (Hunt et al., 2006, 2011). Nonsignificant increases in HFO power were also observed following ketamine and PCP treatment. Changes in lower frequency bands in the striatum failed to reach significance though decreases in those frequency bands were expected (Dimpfel and Spüler, 1990).

In the hippocampus, significant decreases in delta and alpha power following ketamine administration were found in the amperometric signal. MK-801 also induced a significant increase in hippocampal theta, suggesting a peak shift down from alpha when non-competitively blocking NMDA-Rs. A decrease in delta (Dimpfel and Spüler, 1990) and theta power (Dimpfel and Spüler, 1990; Pitkänen et al., 1995; Ehrlichman et al., 2009; Lazarewicz et al., 2010; Kittelberger et al., 2012) was expected; though for hippocampal theta some groups have reported no change (Leung, 1985; Dimpfel and Spüler, 1990; Ehlers et al., 1992; Ma and Leung, 2000; Ehrlichman et al., 2009; Kittelberger et al., 2012; Saunders et al., 2012) or an increase (Dimpfel and Spüler, 1990) following treatment with non-competitive NMDA-R antagonists. These inconsistencies may be explained along three lines of reasoning: 1) Different drugs and doses appear to modulate these frequency bands in different ways; 2) some studies measure LFP from the whole hippocampus and others from specific hippocampal subfields; and 3) lack of agreement about theta's frequency range is common with most papers defining theta as $5-10 \mathrm{~Hz}$ as opposed to splitting that range into $5-8 \mathrm{~Hz}$ for theta and $9-12 \mathrm{~Hz}$ for alpha. However, the case for hippocampal gamma and HFO is more clearcut with the expected increase in the former and no change in the latter found following MK-801 treatment (with non-significant increases observed following the other two drug treatments), contrary to the case in the striatum (Ma and Leung, 2000; Ehrlichman et al., 2009; Lazarewicz et al., 2010; Hunt et al., 2011; Saunders et al., 2012).

Overall, these changes largely follow the trajectories predicted by the electrophysiological literature. The amperometric data confirms that there are clear differences between the striatum and hippocampus in how LFP responds to exploratory behaviour and pharmacological intervention. Further evidence to support the hypothesis that LFP is part of the amperometric signal can be gleaned from the movement power spectra with hippocampal power spectra being consistently larger than in the striatum due to the laminar organisation of the hippocampus (Berke, 2005). Interestingly, there is a less clear distinction between regions based on the $\mathrm{tO}_{2}$ changes with significant increases in both regions observed. Changes in $\mathrm{tO}_{2}$ (and therefore BOLD) does appear to be useful in confirming the time courses for when an animal is moving or when a drug is having an neurological effect as most of the pertinent changes in LFP occur concurrently.

It is currently impossible to differentiate between changes in power associated with $\mathrm{tO}_{2}$ ( $\mathrm{Li}$ et al., 2014) and slow LFP oscillations ( $<1 \mathrm{~Hz}$; Penttonen and Buzsáki, 2003); a second LFP-only recording electrode in close proximity to the CPE would be required to determine any possible relationships between slow oscillatory activity and $\mathrm{tO}_{2}$ levels. However, given that the power spectra obtained from recordings made at different applied voltages and from recordings made by using the CPEs in place of traditional electrophysiological recording electrodes are so similar, it is possible to say 
that the higher frequency components of the amperometric signal are due to changes in the LFP (Zhang et al., 2009). This allows for one set of electrodes to be used to simultaneously monitor changes in $\mathrm{tO}_{2}$, a BOLD-like measurement (Lowry et al., 2010), with LFP, an electrophysiological biomarker that can also be measured in humans. As up to four electrodes can be implanted simultaneously, it is possible to dissect functional dissociation between regions based on pharmacological interventions and behavioural processes with these biomarkers. This technique consequently lends itself to drug evaluation trials by providing chronic, real-time information that can be used to better understand the mechanisms and validity of novel and established therapeutics with direct comparisons to neuroimaging data from human trials.

\section{Acknowledgements}

The research was funded by Science Foundation Ireland (12/ TIDA/I2308) and the Irish Research Council (GOIPD/2013/420).

\section{References}

Berke, J.D., 2005. Participation of striatal neurons in large-scale oscillatory networks. In: Bolam, J.P., Ingham, C.A., Magill, P.J. (Eds.), The Basal Ganglia VIII. Springer, New York, pp. 25-35.

Bolger, F.B., Bennett, R., Lowry, J.P., 2011a. An in vitro characterisation comparing carbon paste and Pt microelectrodes for real-time detection of brain tissue oxygen. Analyst 136, 4028-4035.

Bolger, F.B., Lowry, J.P., 2005. Brain tissue oxygen: in vivo monitoring with carbon paste electrodes. Sensors 5, 473-487.

Bolger, F.B., McHugh, S.B., Bennett, R., Li, J., Ishiwari, K., Francois, J., et al., 2011b. Characterisation of carbon paste electrodes for real-time amperometric monitoring of brain tissue oxygen. J. Neurosci. Methods 195, 135-142.

Broberg, B.V., Madsen, K.H., Plath, N., Olsen, C.K., Glenthøj, B.Y., Paulson, O.B., et al., 2013. A schizophrenia rat model induced by early postnatal phencyclidine treatment and characterized by Magnetic Resonance Imaging. Behav. Brain Res. $250,1-8$.

Chin, C.L., Upadhyay, J., Marek, G.J., Baker, S.J., Zhang, M., Mezler, M., et al., 2011. Awake rat pharmacological magnetic resonance imaging as a translational pharmacodynamic biomarker: metabotropic glutamate $2 / 3$ agonist modulation of ketamine-induced blood oxygenation level dependence signals. J. Pharmacol. Exp. Ther. 336, 709-715.

Dimpfel, W., Spüler, M., 1990. Dizocilpine (MK-801), ketamine and phencyclidine: low doses affect brain field potentials in the freely moving rat in the same way as activation of dopaminergic transmission. Psychopharmacol. Berl. 101, 317-323.

Ehlers, C.L., Kaneko, W.M., Wall, T.L., Chaplin, R.I., 1992. Effects of dizocilpine (MK801) and ethanol on the EEG and event-related potentials (ERPS) in rats. Neuropharmacology 31, 369-378.

Ehrlichman, R.S., Gandal, M.J., Maxwell, C.R., Lazarewicz, M.T., Finkel, L.H., Contreras, D., et al., 2009. N-methyl-d-aspartic acid receptor antagonistinduced frequency oscillations in mice recreate pattern of electrophysiological deficits in schizophrenia. Neuroscience 158, 705-712.

Finnerty, N.J., Bolger, F.B., Pålsson, E., Lowry, J.P., 2013. An investigation of hypofrontality in an animal model of schizophrenia using real-time microelectrochemical sensors for glucose, oxygen, and nitric oxide. ACS Chem. Neurosci. 4, 825-831.

Francois, J., Conway, M.W., Lowry, J.P., Tricklebank, M.D., Gilmour, G., 2012. Changes in reward-related signals in the rat nucleus accumbens measured by in vivo oxygen amperometry are consistent with fMRI BOLD responses in man. Neuroimage $60,2169-2181$.

Francois, J., Grimm, O., Schwarz, A.J., Schweiger, J., Haller, L., Risterucci, C., et al., 2016. Ketamine suppresses the ventral striatal response to reward anticipation: a cross-species translational neuroimaging study. Neuropsychopharmacology 41, 1386-1394.

Francois, J., Huxter, J., Conway, M.W., Lowry, J.P., Tricklebank, M.D., Gilmour, G., 2014. Differential contributions of infralimbic prefrontal cortex and nucleus accumbens during reward-based learning and extinction. J. Neurosci. 34, 596-607.

Gozzi, A., Large, C.H., Schwarz, A., Bertani, S., Crestan, V., Bifone, A., 2008a. Differential effects of antipsychotic and glutamatergic agents on the phMRI response to phencyclidine. Neuropsychopharmacology 33, 1690-1703.
Gozzi, A., Schwarz, A., Crestan, V., Bifone, A., 2008b. Drug-anaesthetic interaction in phMRI: the case of the psychotomimetic agent phencyclidine. Magn. Reson Imaging 26, 999-1006.

Hodkinson, D.J., de Groote, C., McKie, S., Deakin, J.F., Williams, S.R., 2012. Differential effects of anaesthesia on the phMRI response to acute ketamine challenge. Br. J. Med. Med. Res. 2, 373-385.

Hunt, M.J., Falinska, M., Łeski, S., Wójcik, D.K., Kasicki, S., 2011. Differential effects produced by ketamine on oscillatory activity recorded in the rat hippocampus, dorsal striatum and nucleus accumbens. J. Psychopharmacol. 25, 808-821.

Hunt, M.J., Kasicki, S., 2013. A systematic review of the effects of NMDA receptor antagonists on oscillatory activity recorded in vivo. J. Psychopharmacol. 27, 972-986.

Hunt, M.J., Raynaud, B., Garcia, R., 2006. Ketamine dose-dependently induces highfrequency oscillations in the nucleus accumbens in freely moving rats. Biol. Psychiatry 60, 1206-1214.

Janhunen, S.K., Svärd, H., Talpos, J., Kumar, G., Steckler, T., Plath, N., et al., 2015. The subchronic phencyclidine rat model: relevance for the assessment of novel therapeutics for cognitive impairment associated with schizophrenia. Psychopharmacol. Berl. 232, 4059-4083.

Kealy, J., Bennett, R., Lowry, J.P., 2013. Simultaneous recording of hippocampal oxygen and glucose in real time using constant potential amperometry in the freely-moving rat. J. Neuro Methods 215, 110-120.

Kealy, J., Bennett, R., Lowry, J.P., 2015. Real-time effects of insulin-induced hypoglycaemia on hippocampal glucose and oxygen. Brain Res. 1598, 76-87.

Kittelberger, K., Hur, E.E., Sazegar, S., Keshavan, V., Kocsis, B., 2012. Comparison of the effects of acute and chronic administration of ketamine on hippocampal oscillations: relevance for the NMDA receptor hypofunction model of schizophrenia. Brain Struct. Funct. 217, 395-409.

Lazarewicz, M.T., Ehrlichman, R.S., Maxwell, C.R., Gandal, M.J., Finkel, L.H., Siegel, S.J., 2010. Ketamine modulates theta and gamma oscillations. J. Cogn. Neurosci. 22, 1452-1464.

Leung, L.W., 1985. Spectral analysis of hippocampal EEG in the freely moving rat: effects of centrally active drugs and relations to evoked potentials. Electroencephalogr. Clin. Neurophysiol. 60, 65-77.

Li, J., Ishiwari, K., Conway, M.W., Francois, J., Huxter, J., Lowry, J.P., et al., 2014 Dissociable effects of antipsychotics on ketamine-induced changes in regional oxygenation and inter-regional coherence of low frequency oxygen fluctuations in the rat. Neuropsychopharmacology 39, 1635-1644.

Littlewood, C.L., Cash, D., Dixon, A.L., Dix, S.L., White, C.T., O'Neill, M.J., et al., 2006. Using the BOLD MR signal to differentiate the stereoisomers of ketamine in the rat. Neuroimage 32, 1733-1746.

Lowry, J.P., Boutelle, M.G., Fillenz, M., 1997. Measurement of brain tissue oxygen at a carbon past electrode can serve as an index of increases in regional cerebral blood flow. J. Neurosci. Methods 71, 177-182.

Lowry, J.P., Boutelle, M.G., O'Neill, R.D., Fillenz, M., 1996. Characterization of carbon paste electrodes in vitro for simultaneous amperometric measurement of changes in oxygen and ascorbic acid concentrations in vivo. Analyst 121 $761-766$.

Lowry, J.P., Griffin, K., McHugh, S.B., Lowe, A.S., Tricklebank, M., Sibson, N.R., 2010 Real-time electrochemical monitoring of brain tissue oxygen: a surrogate for functional magnetic resonance imaging in rodents. Neuroimage 52, 549-555.

Ma, J., Leung, L.S., 2000. Relation between hippocampal gamma waves and behavioral disturbances induced by phencyclidine and methamphetamine. Behav. Brain Res. 111, 1-11.

McHugh, S.B., Fillenz, M., Lowry, J.P., Rawlins, J.N., Bannerman, D.M., 2011. Brain tissue oxygen amperometry in behaving rats demonstrates functional dissociation of dorsal and ventral hippocampus during spatial processing and anxiety Eur. J. Neurosci. 33, 322-337.

Neill, J.C., Harte, M.K., Haddad, P.M., Lydall, E.S., Dwyer, D.M., 2014. Acute and chronic effects of NMDA receptor antagonists in rodents, relevance to negative symptoms of schizophrenia: a translational link to humans. Eur. Neuropsychopharmacol. 24, 822-835.

O'Neill, R.D., Grünewald, R.A., Fillenz, M., Albery, W.J., 1982. Linear sweep voltammetry with carbon paste electrodes in the rat striatum. Neuroscience 7 1945-1954.

Penttonen, M., Buzsáki, G., 2003. Natural logarithmic relationship between brain oscillators. Thalamus Relat. Syst. 2, 145-152.

Pitkänen, M., Sirviö, J., Ylinen, A., Koivisto, E., Sr, Riekkinen P., 1995. Effects of NMDA receptor modulation on hippocampal type 2 theta activity in rats. Gen. Pharmacol. 26, 1065-1070.

Saunders, J.A., Gandal, M.J., Siegel, S.J., 2012. NMDA antagonists recreate signal-tonoise ratio and timing perturbations present in schizophrenia. Neurobiol. Dis. 46, 93-100.

Zhang, H., Lin, S.C., Nicolelis, M.A., 2009. Acquiring local field potential information from amperometric neurochemical recordings. J. Neurosci. Methods 179, 191-200. 